\title{
Effect of particle geometry on phase transitions in two-dimensional liquid crystals
}

\author{
Yuri Martínez-Ratón ${ }^{\text {a) }}$ \\ Grupo Interdisciplinar de Sistemas Complejos (GISC), Departamento de Matemáticas, Escuela Politécnica \\ Superior, Universidad Carlos III de Madrid, Avenida de la Universidad 30, E-28911 Leganés, \\ Madrid, Spain \\ Enrique Velasco ${ }^{\text {b) }}$ \\ Departamento de Física Teórica de la Materia Condensada and Instituto de Ciencia de Materiales Nicolás \\ Cabrera, Universidad Autónoma de Madrid, E-28049 Madrid, Spain \\ Luis Mederos ${ }^{\mathrm{c})}$ \\ Instituto de Ciencia de Materiales, Consejo Superior de Investigaciones Científicas, E-28049 Cantoblanco, \\ Madrid, Spain
}

(Received 21 October 2004; accepted 24 November 2004; published online 26 January 2005)

\begin{abstract}
Using a version of density-functional theory which combines Onsager approximation and fundamental-measure theory for spatially nonuniform phases, we have studied the phase diagram of freely rotating hard rectangles and hard discorectangles. We find profound differences in the phase behavior of these models, which can be attributed to their different packing properties. Interestingly, bimodal orientational distribution functions are found in the nematic phase of hard rectangles, which cause a certain degree of biaxial order, albeit metastable with respect to spatially ordered phases. This feature is absent in discorectangles, which always show unimodal behavior. This result may be relevant in the light of recent experimental results which have confirmed the existence of biaxial phases. We expect that some perturbation of the particle shapes (either a certain degree of polydispersity or even bimodal dispersity in the aspect ratios) may actually destabilize spatially ordered phases thereby stabilizing the biaxial phase. (c) 2005 American Institute of Physics.
\end{abstract}

[DOI: $10.1063 / 1.1849159$ ]

\section{INTRODUCTION}

Biaxial liquid-crystalline phases were hypothesized in 1970 by Freiser. ${ }^{1}$ Since then many theoretical models have described the conditions that promote biaxial order. However, the experimental search for biaxial phases has been unfruitful until very recently, when Madsen et $a l^{2}$ and Acharya, Primak, and $\mathrm{Kumar}^{3}$ presented convincing evidence for the existence of biaxial order in a fluid composed of boomerang-shaped molecules. In previous work Schlacken, Mogel, and Schiller ${ }^{4}$ analyzed the effect of particle shape on the orientational properties of two-dimensional fluids using scaled-particle theory (SPT). They considered hard ellipses and hard rectangles (HR) and, for the latter, located a spinodal which corresponds to a tetratic phase, where the fluid has two nematic directors oriented perpendicular to each other. Simulation works have been performed by Cuesta and Frenkel ${ }^{5}$ on hard ellipses and by Bates and Frenkel $^{6}$ who focused on hard discorectangles (HDR) and the character of the orientational transition from the isotropic phase. Also Lagomarsino, Dogterom, and Dijkstra ${ }^{7}$ found similar behavior in a fluid of hard spherocylinders confined between two parallel walls which, in the limit of small wall separation, can be considered as a realization of a two-

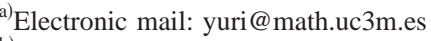

${ }^{b)}$ Electronic mail: enrique.velasco@uam.es

${ }^{c)}$ Electronic mail: 1.mederos@icmm.csic.es
}

dimensional nematic. These simulations have shown that the character of orientational transitions depends strongly on the particle shape: whereas for hard discorectangles the isotropic-nematic transition is of the Kosterlitz-Thouless (KT) type ${ }^{8}$ in the whole aspect ratio regime where the nematic phase is stable against the solid phase, ${ }^{6}$ for hard ellipses a tricritical point separates regions of continuous from firstorder orientational transition. ${ }^{5}$

The density-functional (DF) formalism has been proven to be a powerful tool in the study of bulk nonuniform phases of liquid crystals in three dimensions, specifically for the model of freely rotating hard spherocylinders. ${ }^{9-14}$ To our knowledge, in two dimensions the DF formalism has been applied only to the study of uniform liquid-crystalline phases. In the present work we will not focus on questions related to the KT nature of the isotropic-nematic phase transition, but rather we investigate by DF theory the occurrence of biaxial nematic order in fluids and how this feature depends on the geometry of the particles; we consider both HR and HDR as interaction models. Also we apply DF theory to the study of spinodal instabilities to nonuniform phases in order to analyze the relative stability of these biaxial phases against spatially ordered phases.

The results from the theory allow us to conclude that a certain degree of biaxiality is present in the case of HR. This is truly remarkable, since this fourfold order arises in a one component system of particles with twofold symmetry. How- 
ever the bifurcation analysis using the DF theory allow us to conclude that the tetratic phase is metastable with respect to the solid phase. The remainder of the paper is organized as follows: In Sec. II we introduce the theoretical models for both the uniform phases (SPT in Sec. II A) and for the nonuniform ones (DF in Sec. II B). Section II C is devoted to the bifurcation analysis. Results are shown in Sec. III while some conclusions are drawn in Sec. IV.

\section{MODEL}

The DF approximation proposed is based on SPT, which has proved to be a generic theory which is easily extended to any convex particle geometry. ${ }^{15,16}$ Two extensions, suitable for nonuniform phases, are proposed. Both tend to the SPT in the limit of uniform phases and take into account ideas from fundamental-measure theory (FMT). This theory has been worked out for a system of hard parallelepipeds in the restricted orientations approximation (the so-called Zwanzig model $^{17}$ ) and the corresponding phase diagram, including all nonuniform phases, was recently calculated. ${ }^{18}$ One of the proposed extensions recovers the Onsager second-order virial theory in the low-density limit. The other version is built so as to recover the FMT for parallel hard rectangles, i.e., restricting the particle orientations to point along the nematic director. The construction of a functional which interpolates between the Onsager limit and the high density one using FMT for hard spheres has been proposed by Cinacchi and Schmid for a system of hard spherocylinders in three dimensions. ${ }^{19}$ Nevertheless, their proposal seems to be numerically intractable for nonuniform phases. Here we are interested in constructing a workable density functional.

\section{A. Uniform phases: Scaled particle theory}

The radically different orientational ordering properties shown by the two particle models, HDR and HR (see Fig. 1), can be understood in the framework of SPT. A brief discussion of this theory, as applied to our model systems, follows.

The key quantity to derive the theory is the excluded volume between fluid particles of length $L(\mathrm{HR})$ or $L+\sigma$ (HDR) and width $\sigma$, and a test scaled particle of dimension $L_{s}$ and $\sigma_{s}$ with fixed orientation $\hat{\mathbf{u}}_{1}\left(\phi_{1}\right)$, where $\phi_{1}$ is the angle between the uniaxial axis and the nematic director. The excluded volume is to be averaged over all possible orientations of the fluid particles,

$$
\left\langle V_{\mathrm{excl}}^{\alpha}\right\rangle\left(L_{s}, \sigma_{s}, \phi_{1}\right)=\int d \phi_{2} h\left(\phi_{2}\right) V_{\mathrm{excl}}^{\alpha}\left(L_{s}, \sigma_{s}, \phi_{12}\right),
$$

where $h(\phi)$ is the one-particle orientational distribution function, $\phi_{12}=\phi_{1}-\phi_{2}$, and $V_{\text {excl }}^{\alpha}$ is the excluded volume between two particles which, for rectangles, has the form

$$
\begin{aligned}
& V_{\text {excl }}^{\mathrm{HR}}\left(L_{s}, \sigma_{s}, \phi_{12}\right)=\left(L L_{s}+\sigma \sigma_{s}\right)\left|\sin \phi_{12}\right|+\mathcal{L}_{L} \mathcal{L}_{\sigma}, \\
& \mathcal{L}_{\lambda}=\sqrt{\lambda^{2}+\lambda_{s}^{2}+2 \lambda \lambda_{s}\left|\cos \phi_{12}\right|}
\end{aligned}
$$

with $\lambda=\{L, \sigma\}$, whereas for discorectangles
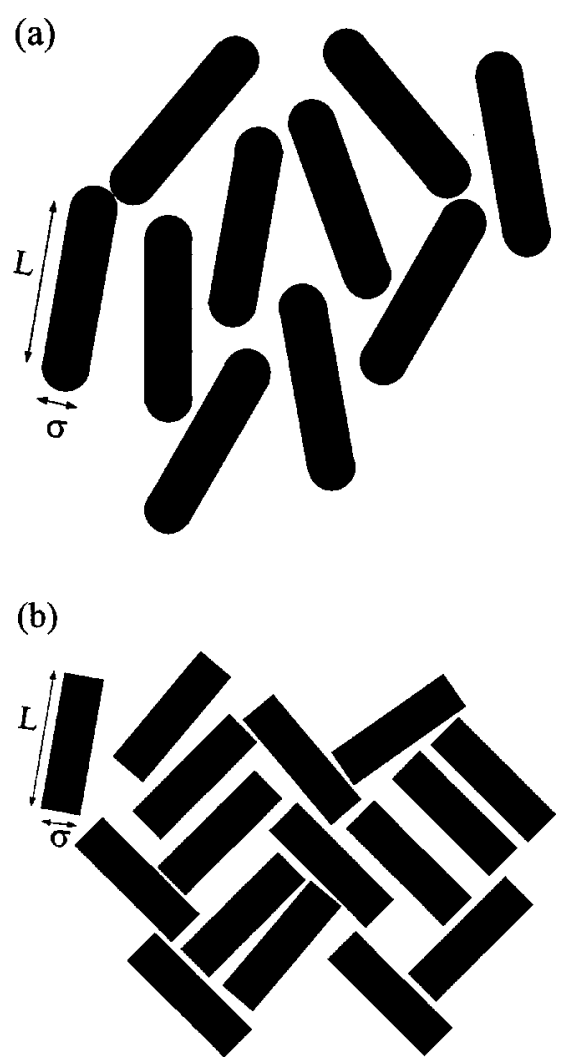

FIG. 1. A system of (a) hard discorectangles in a nematic phase and (b) hard rectangles in a tetratic phase.

$$
\begin{aligned}
V_{\mathrm{excl}}^{\mathrm{HDR}}\left(L_{s}, \sigma_{s}, \phi_{12}\right)= & \left(L+L_{s}\right)\left(\sigma+\sigma_{s}\right)+\frac{\pi}{4}\left(\sigma+\sigma_{s}\right)^{2} \\
& +L L_{s}\left|\sin \phi_{12}\right| .
\end{aligned}
$$

The reversible work required to insert the scaled particle with fixed orientation (which coincides with the excess chemical potential) is, in the limit of small $\operatorname{sizes}^{20}\left(L_{s}, \sigma_{s}\right)$ $\ll(L, \sigma)$ :

$$
\beta \mu_{\mathrm{exc}}\left(\phi_{1}\right) \sim \mu^{(0)}\left(L_{s}, \sigma_{s}, \phi_{1}\right)=-\ln \left[1-\rho\left\langle V_{\mathrm{excl}}\right\rangle\left(L_{s}, \sigma_{s}, \phi_{1}\right)\right],
$$

where $\rho$ is the fluid density. In the opposite limit of large sizes $\left(L_{s}, \sigma_{s}\right) \gg(L, \sigma)$, this work coincides with the thermodynamic reversible work necessary to open a cavity of volume equal to the scaled-particle volume $v_{s}$, which is equal to $P v_{s}, P$ being the fluid pressure. SPT interpolates between these two limits using a Taylor expansion around $\left(L_{s}, \sigma_{s}\right)$ $=(0,0)$, where the second-order term is $P v_{s}$ and, finally, the sizes are taken to be those of the fluid particles, which results in

$$
\beta \mu_{\mathrm{exc}}\left(\phi_{1}\right)=-\ln (1-\eta)+\frac{\rho}{1-\eta} \int d \phi_{2} h\left(\phi_{2}\right) V^{(0)}\left(\phi_{12}\right)+\beta P v
$$

where $\eta=\rho v$ is the packing fraction and $V^{(0)}$ $=V_{\text {excl }}\left(L, \sigma, \phi_{12}\right)-2 v$, with $v$ the particle volume. The excess chemical potential of the fluid is the angular average 


$$
\begin{aligned}
\beta \mu_{\mathrm{exc}}= & \int d \phi_{1} h\left(\phi_{1}\right)\left[\beta \mu_{\mathrm{exc}}\left(\phi_{1}\right)\right]=-\ln (1-\eta)+\frac{2 \eta}{1-\eta} S_{0} \\
& +\beta P v,
\end{aligned}
$$

where we have defined $S_{0}=\left\langle\left\langle V^{(0)}\right\rangle\right\rangle /(2 v)$ and $\langle\langle\cdots\rangle\rangle$ is the double angular average. Using the definitions of the excess chemical potential and pressure in terms of the excess free energy density $\Phi_{\text {exc }}$ a differential equation for $\varphi_{\mathrm{exc}}=\Phi_{\mathrm{exc}} v$ is obtained, with solution

$$
\varphi_{\mathrm{exc}}=\eta\left[-\ln (1-\eta)+\frac{\eta}{1-\eta} S_{0}\right]
$$

and, correspondingly, the pressure is given by

$$
\beta P v=\frac{\eta}{1-\eta}+\frac{\eta^{2}}{(1-\eta)^{2}} S_{0}
$$

which defines the thermodynamics of the fluid.

In order to proceed we have to specify a functional form for the orientational distribution function, which is taken to be a truncated Fourier expansion. In view of the symmetry $h(\phi)=h(\pi-\phi)$, only even harmonic terms have to be included:

$$
h(\phi)=\frac{1}{\pi}\left(1+\sum_{k \geqslant 1}^{n} h_{k} \cos (2 k \phi)\right) .
$$

Note that $\int_{0}^{\pi} d \phi h(\phi)=1$. The cutoff index $n$ is chosen to guarantee a small enough value $\left|h_{n}\right|<10^{-7}$. With this choice the double angular average $S_{0}$ for hard rectangles becomes

$$
S_{0}=\frac{1}{\pi}\left(\kappa^{1 / 2}+\kappa^{-1 / 2}\right)^{2}\left[1-\frac{1}{2} \sum_{k \geqslant 1}^{n} g_{k} h_{k}^{2}\right],
$$

where

$$
\begin{aligned}
& g_{k}=\left(\frac{\kappa-1}{\kappa+1}\right)^{2}\left(4 k^{2}-1\right)^{-1}, \quad k=2 j+1, \\
& g_{k}=\left(4 k^{2}-1\right)^{-1}, \quad k=2 j .
\end{aligned}
$$

We have defined $\kappa \equiv L / \sigma$ (the aspect ratio). The equilibrium orientational structure of the fluid is obtained by minimizing the free energy per particle, whose ideal part reads

$$
\frac{\varphi_{\text {id }}}{\eta}=\ln \eta-1+\int_{0}^{\pi} d \phi h(\phi) \ln [\pi h(\phi)],
$$

whereas the excess part $\varphi_{\mathrm{exc}} / \eta$ is obtained from Eq. (8). A bifurcation analysis for small values of the $h_{k}$ coefficients leads to the following expression for the difference between free energies $\Delta \varphi=\varphi_{N}-\varphi_{I}$ of nematic $(N)$ and isotropic $(I)$ phases:

$$
\frac{\Delta \varphi}{\eta} \approx\left(1-\frac{2}{3 \pi} y \kappa_{-}^{2}\right) \frac{h_{1}^{2}}{4}+\left(1-\frac{2}{15 \pi} y \kappa_{+}^{2}\right) \frac{h_{2}^{2}}{4},
$$

where $\kappa_{ \pm}=\kappa^{1 / 2} \pm \kappa^{-1 / 2}$ and $y=\eta /(1-\eta)$. Note that the first term is dominant with respect to the second. The isotropic phase gets unstable with respect to the uniaxial nematic phase, $N_{u},\left(h_{1} \neq 0\right)$ when the following condition holds: 1 $-(2 / 3 \pi) y \kappa_{-}^{2}=0$. Another possibility is that the isotropic phase gets unstable when $h_{1}=0, h_{2} \neq 0$, and $1-(2 / 15 \pi) y \kappa_{+}^{2}$ $=0$. This bifurcation corresponds to a phase with $D_{4 h}$ symmetry, which we call tetratic, $N_{t}$. The above conditions occur for the following values of the packing fractions:

$$
\eta_{N_{u}}=\left(1+\frac{2}{3 \pi} \kappa_{-}^{2}\right)^{-1}, \quad \eta_{N_{t}}=\left(1+\frac{2}{15 \pi} \kappa_{+}^{2}\right)^{-1} .
$$

The equality of these two values gives a critical aspect ratio $\kappa^{*}=(3+\sqrt{5}) / 2$ such that, when $\kappa<\kappa^{*}$, the $N_{t}$ phase preempts the $N_{u}$ phase.

In order to calculate the complete phase diagram for uniform phases we minimized the full free energy per particle for each $\kappa$ with respect to the $n$ coefficients $h_{k}$. A commontangent construction was used to locate coexistence boundaries in the case of first-order transitions. To characterize the orientational order we have used the following order parameters:

$$
q_{k}=\int_{0}^{\pi} h(\phi) \cos (2 k \phi) d \phi, \quad k=1,2 .
$$

\section{B. Density-functional theory for nonuniform phases}

Two different approximations are being proposed for the excess part of free energy density $\Phi_{\text {exc }}(\mathbf{r})$ which defines the interaction part of the free energy functional as $\beta \mathcal{F}_{\text {exc }}$ $=\int d \mathbf{r} \Phi_{\text {exc }}(\mathbf{r})$. The first one will later be used to calculate the spinodal instabilities of the HR fluid against nonuniform phases.

As an important ingredient of any density-functional approximation for freely rotating anisotropic particles, we need to impose the Onsager form for the excess part of the freeenergy density in the low density limit:

$$
\begin{aligned}
\Phi_{\mathrm{exc}}^{\mathrm{ONS}}\left(\mathbf{r}_{1}\right)= & -\frac{1}{2} \int d \mathbf{r}_{2} \int d \phi_{1} \int d \phi_{2} \rho\left(\mathbf{r}_{1}, \phi_{1}\right) \rho\left(\mathbf{r}_{2}, \phi_{2}\right) \\
& \times f\left(\mathbf{r}_{1}, \mathbf{r}_{2}, \phi_{1}, \phi_{2}\right),
\end{aligned}
$$

where $f\left(\mathbf{r}_{1}, \mathbf{r}_{2}, \phi_{1}, \phi_{2}\right)$ is the Mayer function between two particles with orientations $\phi_{1}$ and $\phi_{2}$. Another important feature is that the functional capture correctly the high-density limit. For this purpose we will use the FMT to include the remaining part of the density dependence and ensure, in this way, the high-density limit. This dependence enters through the weighted densities $n_{\alpha}(\mathbf{r}) .{ }^{17}$ Finally we impose that the scaled particle theory be recovered in the uniform limit.

As a first proposal we write the following form for the excess part of the free-energy density:

$$
\begin{aligned}
\Phi_{\mathrm{exc}}(\mathbf{r})= & -n_{0}(\mathbf{r}) \ln \left[1-n_{2}(\mathbf{r})\right]-\frac{n_{0}(\mathbf{r}) n_{2}(\mathbf{r})}{1-n_{2}(\mathbf{r})} \\
& +\left[\left(1-n_{2}\right)^{-1} * \omega_{y}^{(0)}\right](\mathbf{r}) \Phi_{\mathrm{exc}}^{\mathrm{ONS}}(\mathbf{r}),
\end{aligned}
$$

where the $n_{\alpha}$ 's are defined as $n_{\alpha}(\mathbf{r})=\left[\rho^{*} \omega_{y}^{(\alpha)}\right](\mathbf{r})$, i.e., they are convolutions of the averaged density profile of rectangles, $\rho(\mathbf{r})=\int d \phi \rho(\mathbf{r}, \phi)$, with some weighting functions given by the expressions 


$$
\begin{aligned}
& \omega_{y}^{(0)}(\mathbf{r})=\frac{1}{4} \delta\left(\frac{\sigma}{2}-|x|\right) \delta\left(\frac{L}{2}-|y|\right), \\
& \omega_{y}^{(2)}(\mathbf{r})=\Theta\left(\frac{\sigma}{2}-|x|\right) \Theta\left(\frac{L}{2}-|y|\right),
\end{aligned}
$$

where $\delta(x)$ and $\Theta(x)$ are the Dirac delta function and the Heaviside function, respectively. An important property of the above proposal is that it recovers the SPT theory in the uniform limit. Note that weighting functions are the characteristic functions which define the support of the particle geometry (a rectangle parallel to the $y$ axis) and its total surface area ${ }^{17}$ (their integrals are two of the fundamental measures of the particles). Note also that, in order to numerically implement the theory, the simplest choice is to take a system of parallel HR as a reference system. Another possibility is to use as a reference system a fluid of parallel hard squares with particles having the same volume as that of the rectangles. This choice may be more adequate to describe isotropic nonuniform phases such as the plastic solid (for which the lattice period is the same in the $x$ and $y$ directions), whereas the former choice should be better for the oriented solid phases. Both reference systems will later be used to calculate the spinodal instabilities to nonuniform phases.

A possible improvement of the theory would be to get rid of the reference system of parallel particles and generalize the definitions of weighting functions (19) and (20) to describe freely rotating particles:

$$
\begin{aligned}
& \omega_{y}^{(0)}(\mathbf{r}, \phi)=\frac{1}{4} \delta\left(\frac{\sigma}{2}-\left|X_{\phi}\right|\right) \delta\left(\frac{L}{2}-\left|Y_{\phi}\right|\right), \\
& \omega_{y}^{(2)}(\mathbf{r}, \phi)=\Theta\left(\frac{\sigma}{2}-\left|X_{\phi}\right|\right) \Theta\left(\frac{L}{2}-\left|Y_{\phi}\right|\right),
\end{aligned}
$$

where $X_{\phi}=x \cos \phi+y \sin \phi$ and $Y_{\phi}=y \cos \phi-x \sin \phi$ are the new Cartesian coordinates in a system rotated by the local angle $\phi$ of the particle, measured with respect to the nematic director (the $y$ axis). Thus the weighted densities $n_{\alpha}$ 's are now calculated from

$$
n_{\alpha}(\mathbf{r})=\int d \mathbf{r}^{\prime} \int d \phi^{\prime} \rho\left(\mathbf{r}+\mathbf{r}^{\prime}, \phi^{\prime}\right) \omega_{y}^{(\alpha)}\left(\mathbf{r}^{\prime}, \phi^{\prime}\right) .
$$

The new expression for $\Phi_{\text {exc }}$ is now given by Eq. (18) where the last term is substituted by

$$
\begin{array}{r}
-\frac{1}{2} \int d \phi_{1}\left[\left(1-n_{2}\right)^{-1} * \omega_{y}^{(0)}\right]\left(\mathbf{r}_{1}, \phi_{1}\right) \rho\left(\mathbf{r}_{1}, \phi_{1}\right) \\
\quad \times \int d \mathbf{r}_{2} \int d \phi_{2} \rho\left(\mathbf{r}_{2}, \phi_{2}\right) f\left(\mathbf{r}_{1}, \mathbf{r}_{2}, \phi_{1}, \phi_{2}\right) .
\end{array}
$$

This term has to be modified due to the fact that the term $\left(1-n_{2}\right)^{-1}$ is now convoluted with the weight $\omega_{y}^{(0)}(\mathbf{r}, \phi)$, which explicitly depends on $\phi$. This approach will not be pursued any further and we leave it for future work.

As can be seen the prefactor of the function $\Phi_{\mathrm{exc}}^{\mathrm{ONS}}$ in Eq. (18) depends on the density profile defined in a rectangular region centered at $\mathbf{r}$ with dimensions $(2 L) \times(2 \sigma)$. This is not the usual case in the formulation of the FMT from first prin-
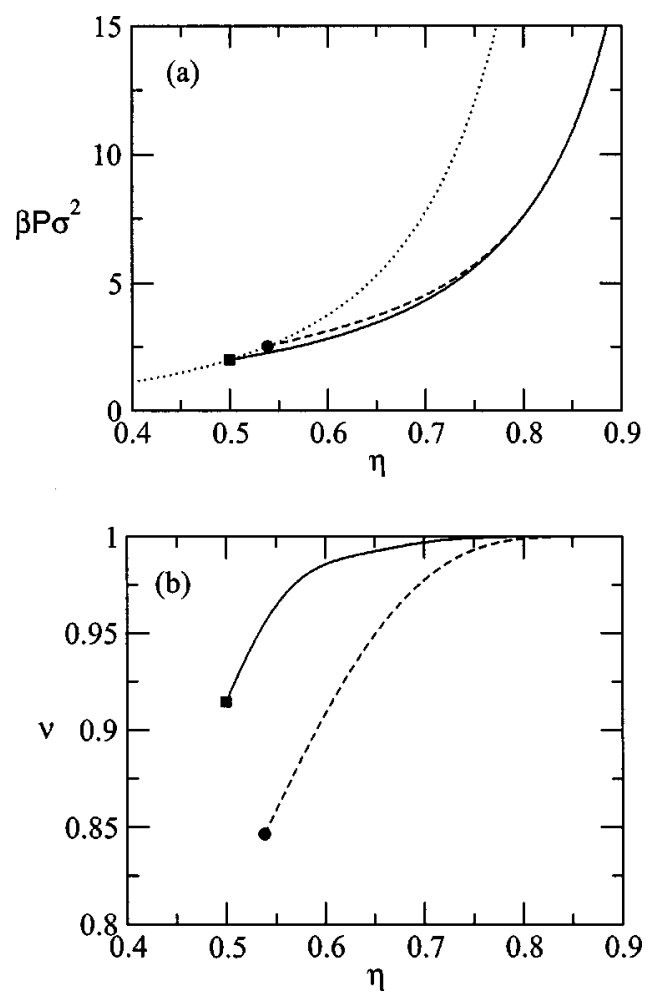

FIG. 2. (a) Equations of state for PHSs as obtained from FMT and the proposed version of DF. Dotted line, fluid branch; dashed line, solid branch from FMT; and solid line, solid branch from the proposed version. The full square and the circle indicate the corresponding bifurcation points. (b) The occupancy probability vs packing fraction along the solid branches. Dashed line, FMT and solid line, present theory.

ciples, but it is necessary because the term $\Phi_{\mathrm{exc}}^{\mathrm{ONS}}$ depends on the Mayer functions which have the range of the excluded surface area.

In order to test the performance of this version of DF we will apply it to the study of freezing in a parallel hard-square (PHS) fluid and compare the results with the FMT derived in Ref. 17. This test is motivated by the fact that the close packing limit is easier to reach in this system compared with the freely rotating case and thus the possible divergences inherent to the particular functional structure can be more easily elucidated.

The density profile of the solid phase was parametrized using Gaussian peaks centered at the sites of a square lattice and the fraction of vacancies was included through a normalization factor in the profile. The minimization of the functional was carried out with respect to the Gaussian width and occupancy probability $\nu$ (one minus the fraction of vacancies). The results are shown in Fig. 2 where the fluid and solid equations of state [Fig. 2(a)] and the occupancy probability vs packing fraction [Fig. 2(b)] are plotted. Comparison is made between results from the present theory and FMT. The fluid branch is the same because, as already pointed out, our theory recovers SPT. Note that the new version stabilizes the solid phase at a lower packing fraction $\eta$ $\approx 0.50$ with a fraction of vacancies $1-\nu=0.085$ to be compared with the FMT result $(\eta \approx 0.53$ and $1-\nu=0.154)$. The lattice parameter $d$ of the solid phase at the bifurcation point, as calculated from the mean density $\rho=\nu / d^{2}$, is equal to 
1.353 from the present version and 1.254 from FMT. All of these results indicate that both functionals produce similar results and, what is more important, the new functional is regular for this kind of density profiles (note that a free minimization would be required to completely settle this point).

Another possibility to construct a DF for freely rotating hard rectangles is to impose that this functional recover the fundamental measure functional (FMF) when the density profile is chosen to have the form $\rho(\mathbf{r}, \phi)=\rho_{y}(\mathbf{r}) \delta(\phi)$, i.e., the system of parallel hard rectangles. Note that, in this limit, we should recover the equation of state of the solid corresponding to a system of parallel hard squares, shown in Fig. 2(a). A free-energy density that conforms to this criterion is proposed in the Appendix.

\section{Bifurcation analysis of nonuniform phases}

At fixed chemical potential $\mu_{0}$ corresponding to a nematic phase characterized by the one particle distribution function $\rho_{0} h(\phi)$, the minimization of the grand potential leads to

$$
\rho(\mathbf{r}, \phi)=\rho_{0} h(\phi) \exp \left[-\beta\left(\frac{\delta \mathcal{F}_{\text {exc }}}{\delta \rho(\mathbf{r}, \phi)}-\mu_{0}\right)\right] .
$$

Assuming a density profile of the form $\rho(\mathbf{r}, \phi)=\rho_{0} h(\phi)$ $+\xi(\mathbf{r}, \phi)$ for the incipient nonuniform phase which bifurcates from the nematic phase, a Taylor expansion of Eq. (25) up to first order in $\xi$ gives

$$
\begin{aligned}
\rho(\mathbf{r}, \phi)= & \rho_{0} h(\phi)\left[1+\int d \mathbf{r}^{\prime} \int d \phi^{\prime} C\left(\mathbf{r}-\mathbf{r}^{\prime}, \phi, \phi^{\prime}\right)\right. \\
& \left.\times \xi\left(\mathbf{r}^{\prime}, \phi^{\prime}\right)\right]
\end{aligned}
$$

where $C\left(\mathbf{r}-\mathbf{r}^{\prime}, \phi, \phi^{\prime}\right)$ is the direct correlation function of the nematic fluid calculated as minus the second functional derivative of $\beta \mathcal{F}_{\text {exc }}$ with respect to the density profile evaluated at $\xi=0$. Equation (26) can be rewritten in Fourier space as

$$
\hat{\xi}(\mathbf{q}, \phi)-\rho_{0} h(\phi) \int d \phi^{\prime} \hat{C}\left(\mathbf{q}, \phi, \phi^{\prime}\right) \hat{\xi}\left(\mathbf{q}, \phi^{\prime}\right)=0,
$$

where the hats over the functions $\xi$ and $C$ indicate Fourier transforms.

Inserting the truncated Fourier expansion of the function $\hat{\xi}(\mathbf{q}, \phi)$,

$$
\hat{\xi}(\mathbf{q}, \phi)=\sum_{k=0}^{n} \xi_{k}(\mathbf{q}) \cos (2 k \phi),
$$

in Eq. (27), multiplying the latter by $\cos (2 j \phi)$, and integrating in $\phi$ from 0 to $2 \pi$ we obtain the following algebraic equation:

$$
\left(I-\rho_{0} T\right) \mathbf{u}=0
$$

where $I$ is the identity matrix, $T$ is a $(n+1) \times(n+1)$ matrix with elements

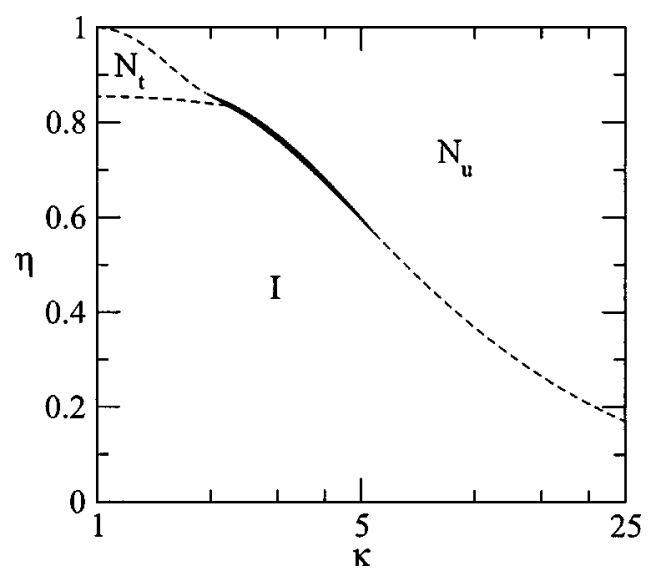

FIG. 3. Phase diagram for hard rectangles in the $\eta-\kappa$ plane. The $\kappa$ axis is represented using a logarithmic scale. Dashed lines indicate second-order transitions, whereas full lines are binodals. The shaded region is the twophase region where phase separation occurs. Labels indicate the three phases involved: isotropic $I$, uniaxial nematic $N_{u}$, and tetratic nematic $N_{t}$.

$$
\begin{aligned}
T_{j k}= & \frac{1}{\pi\left(1+\delta_{j 0}\right)} \int d \phi h(\phi) \\
& \times \int d \phi^{\prime} \cos (2 j \phi) \cos \left(2 k \phi^{\prime}\right) \hat{C}\left(\mathbf{q}, \phi, \phi^{\prime}\right)
\end{aligned}
$$

and $\mathbf{u}=\left[\xi_{0}(\mathbf{q}), \cdots, \xi_{n}(\mathbf{q})\right]^{T}$ is a $n+1$ vector.

The lower value of $\rho_{0}$ and the vector $\mathbf{q}$ for which there exists a nontrivial solution to Eq. (29) can be calculated from

$$
\begin{aligned}
& H\left(\rho_{0}, \mathbf{q}\right) \equiv \operatorname{det}\left(I-\rho_{0} T\right)=0, \\
& \nabla H\left(\rho_{0}, \mathbf{q}\right)=0 .
\end{aligned}
$$

Both equations will be used later to calculate the spinodal instabilities from the isotropic or nematic phases to possible nonuniform phases.

\section{RESULTS}

\section{A. Uniform phases}

In Fig. 3 we show the phase diagram for hard rectangles with $1 \leqslant \kappa \leqslant 25$ as obtained by applying the SPT formalism. For $2.21<\kappa<5.44$ the $I-N_{u}$ transition is of first order. For $\kappa<2.21$ a tetratic nematic phase $N_{t}$ begins to be stable; its stability region is bounded below by a second-order $I-N_{t}$ transition. The region is bounded above by a $N_{t}-N_{u}$ firstorder transition for $1.94<\kappa<2.21$ and by a second-order transition for $1<\kappa<1.94$. This means that there exist two tricritical points: one at $\kappa=5.44$, already predicted by Schlacken, Mogel, and Schiller, ${ }^{4}$ and a second one at $\kappa$ $=1.94$, which can only be predicted by a proper bifurcation analysis from the $N_{t}$ to the $N_{u}$ phases. Figure 4 shows the two-order parameters [Eqs. (16)] along the transition lines to orientationally ordered phases for the systems where these parameters are different from zero. Orientational distribution functions for the $N_{u}$ and $N_{t}$ phases at coexistence for $\kappa=2$ are shown in Fig. 5. Note that the $N_{t}$ phase presents two peaks of the same height (consistent with this phase being invariant with respect to the $D_{4 h}$ symmetry group). By contrast, the $N_{u}$ phase also presents a secondary peak (indicating a certain 


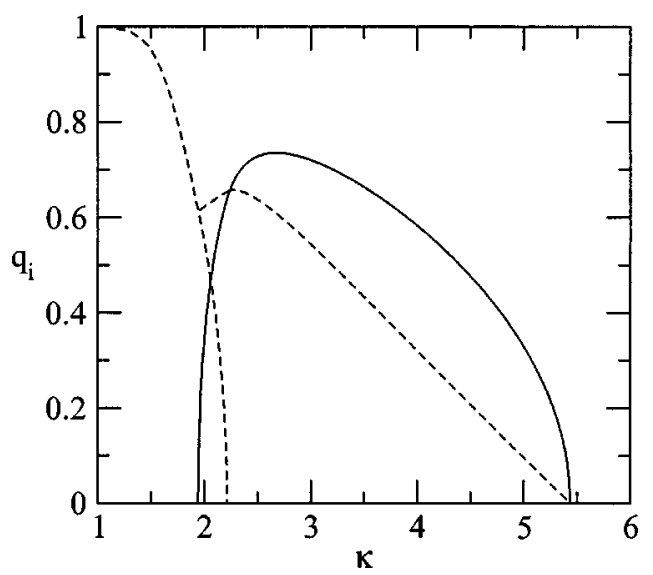

FIG. 4. Order parameters $q_{1}$ and $q_{2}$ along the transition lines to orientationally ordered phases. The full line corresponds to the $q_{1}$ parameter, whereas the dashed lines correspond to the $q_{2}$ parameter.

degree of biaxiality in this phase), but of a smaller height. If $\kappa$ is increased the height of this secondary peak reduces compared to the main peak, eventually disappearing.

We now show the results for hard discorectangles. We have implemented the same calculational scheme and obtained the phase diagram. Only two phases are involved: the isotropic phase $I$ and the uniaxial nematic $N$ phase. The phase boundary $\eta-\kappa$ between the two turns out to be of second order always ${ }^{21}$ and is given by the equation

$$
\eta=\left(1+\frac{2}{3} \frac{(\kappa-1) / \pi}{1+\frac{\pi}{4(\kappa-1)}}\right)^{-1},
$$

where $\kappa=(L+\sigma) / \sigma$ and now $L$ is the length of the rectangular part of the particle and $\sigma$ is its width.

A key difference between hard rectangles and hard discorectangles is that in the former case the excluded volume expression contains a cosine term which is absent in the latter case. This can be seen from Eqs. (2)-(4); substituting $L_{s}=L$ and $\sigma_{s}=\sigma$ we obtain

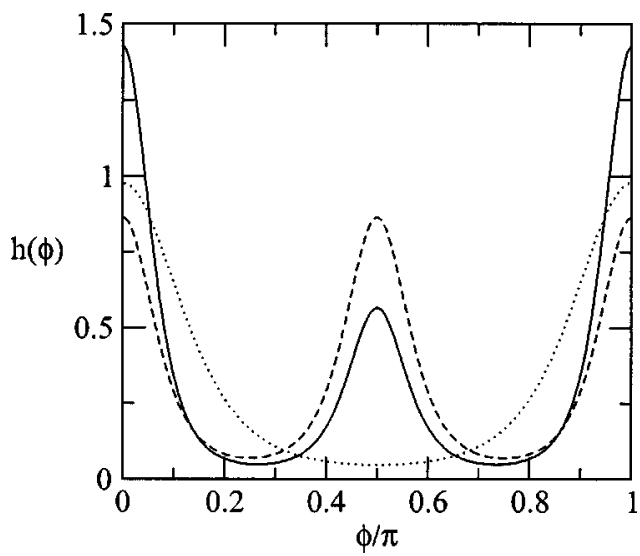

FIG. 5. Angular distribution functions of hard rectangles of the $N_{u}$ (solid line) and $N_{t}$ (dashed line) phases at coexistence and for $\kappa=2$. The dotted line represents the distribution function of hard discorectangles for the same $\kappa$ and for $\eta=0.91$.

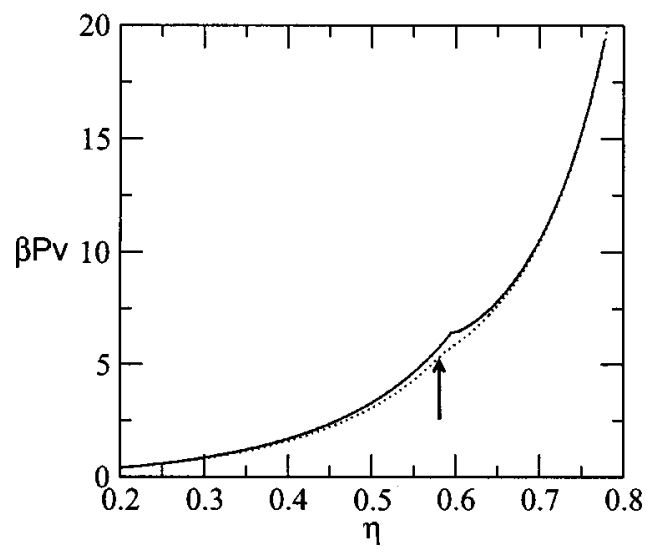

FIG. 6. Equations of state of hard rectangles (solid line) and discorectangles (dotted line) for $\kappa=5$. The arrow indicates the location of the second-order phase transition.

$$
V_{\mathrm{exc}}^{\mathrm{HR}}\left(L, \sigma, \phi_{12}\right)=\left(L^{2}+\sigma^{2}\right)\left|\sin \phi_{12}\right|+2 L \sigma\left(1+\left|\cos \phi_{12}\right|\right),
$$

whereas for discorectangles

$$
V_{\mathrm{exc}}^{\mathrm{HDR}}\left(L, \sigma, \phi_{12}\right)=4 L \sigma+\pi \sigma^{2}+L^{2}\left|\sin \phi_{12}\right| .
$$

This basic difference translates into the $g_{k}$ prefactors in Eq. (11) being different according to the parity of the index for hard rectangles [see Eq. (12)], whereas for hard discorectangles these coefficients have the same form. This feature again shows up in the orientational distribution function which, as pointed out above, exhibits a secondary maximum at $\phi=\pi / 2$ in the case of rectangles, a peak which is absent in the case of discorectangles, see Fig. 5. In this figure we also show the angular distribution function corresponding to the $N$ phase of hard discorectangles for the same $\kappa$ (and for $\eta$ $=0.91$ ).

In order to compare the equations of state of hard rectangles and discorectangles for the same aspect ratio $\kappa=5$, the reduced pressure vs packing fraction is shown in Fig. 6. It can be seen that the two equations of state show a similar behavior, except in the neighborhood of the corresponding phase transitions, which are of first and second order for rectangles and discorectangles, respectively.

\section{B. Nonuniform phases}

To elucidate the absolute stability of the tetratic nematic phase (see Fig. 3), we have carried out a bifurcation analysis following the lines described in Sec. II C, i.e., solving Eqs. (31) and (32) in order to find the values of the packing fraction and wave vector for which the uniform phases destabilize to nonuniform phases. The results are shown in Fig. 7 where we plot all spinodal lines of the $I-N_{u}$, the $I-S$ ( $S$ being a spatially ordered phase, whose structure will require further analysis), and the $N_{u}-S$ transitions. Note that Fig. 7 is in fact the same as Fig. 3, but spinodal lines from the above bifurcation analysis have been superimposed and, at the same time, transition lines in Fig. 3 which turn out to be preempted by nonuniform phases have been dropped.

As can be seen in Fig. 7, for $\kappa \lesssim 5.5$ the $I$ phase destabilizes directly to the $S$ phase, so the tetratic nematic phase is 


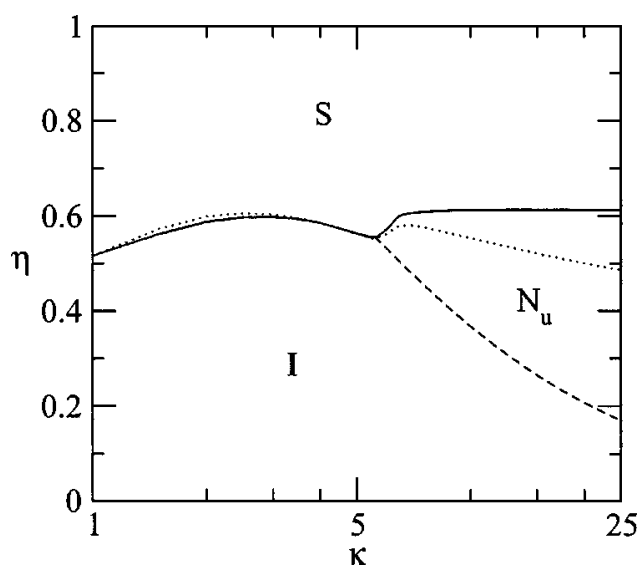

FIG. 7. Phase diagram of hard rectangles including the spinodal lines of the $I-S$ and $N_{u}-S$ transitions. The solid and dotted lines represent the spinodal curves calculated from the functional obtained from Eq. (18), using a reference system of parallel rectangles and parallel squares, respectively. For the latter case the volume of the squares were taken to be the same as that of rectangles.

not stable. Nevertheless, a tetratic order is possible in spatially ordered phases. Taking into account that the $I-S$ transition is probably of first order a complete functional minimization with respect to two dimensional density profiles is required to settle this question. In Fig. 7 the nonuniform phase spinodals are calculated using two reference systems: parallel rectangles and parallel squares. As was discussed in Sec. II B, the last one should better describe the I-S transition, whereas the former should be more appropriate for the $N_{u}-S$ transition. In other words, the dotted line should be more reliable for $\kappa<5.5$, whereas the solid line is expected to be more accurate for $\kappa>5.5$ (note that both reference systems give similar results in the first region and naturally these results coincide exactly at $\kappa=1$ ).

If we use the density functional for freely rotating particles without a reference system of parallel particles (see Sec. II B), the fluid-solid transition is expected to shift to higher densities. The reason for this behavior is that a reference system composed of parallel particles enhances a crystalline local order which is absent in the isotropic phase. Nevertheless, we expect that, within the present version of the theory, the tetratic phase still remains unstable with respect to the solid phase. Calculations using this functional is a task in progress and will allow us to finally settle this question.

\section{CONCLUSIONS}

The main conclusion of the present work is that different particle geometries may account for the different nature of the orientational phase transitions involved (continuous versus first order with the presence of a tricriticcal point). But, even more important, particles possessing uniaxial symmetry (hard rectangles) may lead to a phase with a dramatically different symmetry: the tetratic phase. This finding is based upon minimization of a density-functional theory constructed using ideas from SPT. However, the tetratic phase has been found to be only metastable with respect to a spatially ordered phase. This result was obtained using a bifurcation analysis of a version of density-functional theory proposed in the present paper. The theory combines the Onsager functional with the FMF for hard rectangles. A word of caution is in order here: the proposed functional does not reproduce the exact third virial coefficient, which is expected to be of vital importance at high density, and these neglected correlations should play a role of paramount importance in the stabilization of a phase with tetratic symmetry. It might well be that a full theory, incorporating correlations between three particles, could stabilize the tetratic phase even further and predict it to be stable at densities below that at which crystallization occurs. Nevertheless, it is interesting to note that the present theory already confirms that an oriented fluid of hard rectangles presents some degree of biaxial order for small aspect ratios. The theory could also be used to predict the stability of a number of two-dimensional phases that occur in Langmuir monolayers. $^{22}$

Previous work on polydisperse mixtures of hard spherocylinders, ${ }^{23,24}$ or parallelepipeds ${ }^{25}$ in three dimensions, have shown that polydispersity enhances the stability of the uniform phases with respect to nonuniform phases. While columnar order admits a higher degree of polydispersity, the smectic or solid phases normally have a terminal polydispersity beyond which these phases are no longer stable. ${ }^{23}$ This fact can be easily understood if we take into account that (i) any periodic packing is difficult to attain in a mixture of particles with different characteristic lengths and (ii) mixing increases the ideal mixing entropy of uniform phases and, as a consequence, decreases the Helmholtz free energy. Thus, some degree of polydispersity (either unimodal or bimodal) will certainly increase the density of the transition from the fluid to a spatially ordered phase in a polydisperse mixture of hard rectangles. Is this effect enough to stabilize the tetratic phase? This is an open question that we will try to elucidate in a future work.

\section{ACKNOWLEDGMENTS}

Y.M.-R. was supported by a Ramón y Cajal research contract. This work is part of research Projects Nos. BFM2003-0180, BFM2001-0224-C02-01, BFM2001-0224C02-02, and BFM2001-1679-C03-02 (DGI) of the Ministerio de Educación y Ciencia (Spain).

\section{APPENDIX: ALTERNATIVE VERSION OF DENSITY-FUNCTIONAL}

In this Appendix we present an alternative version of DF which recovers the FMT for parallel HR and SPT in the uniform limit. Our proposal for the excess free-energy density is

$$
\begin{aligned}
\Phi_{\mathrm{exc}}(\mathbf{r})= & -n_{0}(\mathbf{r}) \ln \left[1-n_{2}(\mathbf{r})\right]-\frac{n_{1 x}(\mathbf{r}) n_{1 y}(\mathbf{r})}{1-n_{2}(\mathbf{r})} \\
& -2 \sum_{\mu=x, y}\left[\frac{\partial \Phi_{\mathrm{exc}}^{\mathrm{ref}}}{\partial n_{1 \mu}} \omega_{y}^{(1 \mu)}\right](\mathbf{r}) \\
& \times \frac{\Phi_{\mathrm{exc}}^{\mathrm{ONS}}(\mathbf{r})}{\int d \mathbf{r}^{\prime} \rho\left(\mathbf{r}^{\prime}\right) f_{\mathrm{ref}}\left(\mathbf{r}-\mathbf{r}^{\prime}\right)},
\end{aligned}
$$


where $f_{\text {ref }}(\mathbf{r})=-\Theta(\sigma-|x|) \Theta(L-|y|)$ is the Mayer function of two parallel hard rectangles, $\Phi_{\text {exc }}^{\text {ref }}$ is the excess part of the Helmholtz free-energy density obtained from the FMT for parallel hard rectangles, ${ }^{17}$ i.e.,

$$
\Phi_{\mathrm{exc}}^{\mathrm{ref}}=-n_{0} \ln \left(1-n_{2}\right)+\frac{n_{1 x} n_{1 y}}{1-n_{2}}
$$

while the new weights are

$$
\begin{aligned}
& \omega_{y}^{(1 x)}=\frac{1}{2} \delta\left(\frac{\sigma}{2}-|x|\right) \Theta\left(\frac{L}{2}-|y|\right), \\
& \omega_{y}^{(1 y)}=\frac{1}{2} \Theta\left(\frac{\sigma}{2}-|x|\right) \delta\left(\frac{L}{2}-|y|\right) .
\end{aligned}
$$

Note that the uniform limit of $\Phi_{\text {exc }}(\mathbf{r}) v$ [see Eq. (A1)] coincides with the expression (8) obtained from the SPT.

Now we will show that the free-energy functional obtained from Eq. (A1) reduces to the FMT for the case of parallel rectangles. In this limit it is easily shown that

$$
\frac{\Phi_{\mathrm{exc}}^{\mathrm{ONS}}(\mathbf{r})}{\int d \mathbf{r}^{\prime} \rho\left(\mathbf{r}^{\prime}\right) f_{\mathrm{ref}}\left(\mathbf{r}-\mathbf{r}^{\prime}\right)} \rightarrow-\frac{1}{2} \rho(\mathbf{r}) .
$$

The above limit, together with the fact that

$$
\int d \mathbf{r}\left[\frac{n_{1 \mu}}{1-n_{2}} * \omega_{y}^{(1 \nu)}\right](\mathbf{r}) \rho(\mathbf{r})=\int d \mathbf{r} \frac{n_{1 \mu}(\mathbf{r}) n_{1 \nu}(\mathbf{r})}{1-n_{2}(\mathbf{r})}
$$

(where $\mu \neq \nu$ ) allow us to conclude that

$$
\int d \mathbf{r} \Phi_{\mathrm{exc}}(\mathbf{r}) \rightarrow \int d \mathbf{r} \Phi_{\mathrm{exc}}^{\mathrm{ref}}(\mathbf{r})
$$

${ }^{1}$ M. J. Freiser, Phys. Rev. Lett. 24, 1041 (1970).

${ }^{2}$ L. A. Madsen, T. J. Dingemans, M. Nakata, and E. T. Samulski, Phys. Rev. Lett. 92, 145505 (2004)

${ }^{3}$ B. R. Acharya, A. Primak, and S. Kumar, Phys. Rev. Lett. 92, 145506 (2004).

${ }^{4}$ H. Schlacken, H.-J. Mogel, and P. Schiller, Mol. Phys. 93, 777 (1998).

${ }^{5}$ J. A. Cuesta and D. Frenkel, Phys. Rev. A 42, 2126 (1990).

${ }^{6}$ M. A. Bates and D. Frenkel, J. Chem. Phys. 112, 10034 (2000).

${ }^{7}$ M. C. Lagomarsino, M. Dogterom, and M. Dijkstra, J. Chem. Phys. 119, 3535 (2003).

${ }^{8}$ D. Frenkel and R. Eppenga, Phys. Rev. A 31, 1776 (1985).

${ }^{9}$ A. M. Somoza and P. Tarazona, Phys. Rev. Lett. 61, 2566 (1988); Phys. Rev. A 41, 965 (1990).

${ }^{10}$ A. Poniewierski and R. Holyst, Phys. Rev. Lett. 61, 2461 (1988).

${ }^{11}$ B. Tjipto-Margo and G. Evans, Mol. Phys. 74, 85 (1991).

${ }^{12}$ R. van Roij, P. Bolhuis, B. Mulder, and D. Frenkel, Phys. Rev. E 52, R1277 (1995).

${ }^{13}$ H. Graf and H. Lowen, J. Phys.: Condens. Matter 11, 1435 (1999).

${ }^{14}$ E. Velasco, L. Mederos, and D. E. Sullivan, Phys. Rev. E 62, 3708 (2000).

${ }^{15}$ M. A. Cotter and D. Wacker, Phys. Rev. A 18, 2669 (1978).

${ }^{16}$ B. Barboy and M. Gelbart, J. Chem. Phys. 71, 3053 (1979).

${ }^{17}$ J. A. Cuesta and Y. Martínez-Ratón, Phys. Rev. Lett. 78, 3681 (1997).

${ }^{18}$ Y. Martínez-Ratón, Phys. Rev. E 69, 061712 (2004).

${ }^{19}$ G. Cinacchi and F. Schmid, J. Phys.: Condens. Matter 14, 12223 (2002).

${ }^{20}$ H. Reiss, H. L. Frisch, and J. L. Lebowitz, J. Chem. Phys. 31, 369 (1959).

${ }^{21}$ Note that the general Landau expansion of the free energy in two dimensions contains only powers of $\operatorname{Tr} Q^{2}$, where $Q$ is the tensor order parameter. Depending on the sign of the coefficient associated with the term $\left(\operatorname{Tr} Q^{2}\right)^{2}$, which in turn is determined by the particle geometry, the transition can be of first or second order. By contrast, in three dimensions the transition must be of first order due to the presence of a term $\operatorname{Tr} Q^{3}$.

${ }^{22}$ V. M. Kaganer, H. Möhwald, and P. Dutta, Rev. Mod. Phys. 71, 779 (1999).

${ }^{23}$ J. M. Polson and D. Frenkel, Phys. Rev. E 56, R6260 (1997).

${ }^{24}$ G. Cinacchi, L. Mederos, and E. Velasco, J. Chem. Phys. 121, 3854 (2004).

${ }^{25}$ Y. Martínez-Ratón and J. A. Cuesta, J. Chem. Phys. 118, 10164 (2003). 